Scientific Paper

\title{
Diffuse reflectance spectroscopy for identification of carcinogen transformation stages in skin tissue
}

\author{
Kawthar SHURRAB ${ }^{1, a}$, Nabil KOCHAJI ${ }^{2}$, Wesam BACHIR ${ }^{3}$ \\ ${ }^{I}$ Medical Physics, Biomedical Photonics Laboratory, Higher Institute for Laser Research and Applications, Damascus \\ University, Damascus, Syria \\ ${ }^{2}$ Faculty of Dentistry, Al-Sham University, Damascus, Syria \\ ${ }^{3}$ Biomedical Photonics Laboratory, Department of Laser Physics and Technology, Higher Institute for Laser Research and \\ Applications, Damascus University, Damascus, Syria \\ ${ }^{a}$ E-mail address: sh_kawther@yahoo.com
}

(received 20 September 2018; revised 19 February, 2 April and 7 May 2019; accepted 8 May 2019)

\begin{abstract}
Today, to establish a diagnosis, the patient must undergo a biopsy followed by histopathological diagnosis, which causes unnecessary cost, patient trauma, and time delay to obtain a diagnosis. However, the metastases can be discovered by diffuse reflectance spectroscopy, which is a simple method that investigates the light distribution within tissue. The theme of this paper is the use of diffuse reflectance spectroscopy (DRS) to determine the optical spectrum of hamster specimen's tissue and to differentiate biological changes due to laser irradiation (scattering, and cell changes) under the skin. DRS measurements were made on healthy and malignant tissue to diagnose the stages of cancer formation using a fiber-optic probe. The results show that malignant tissue is characterized by a significant decrease in diffuse reflectance spectrum compared to normal tissue.
\end{abstract}

Key words: optical; spectrum; diffuse reflectance spectroscopy; healthy; malignant tissue.

\section{Introduction}

Knowledge of the degree to which tissue scatters light is important for understanding the physical processes manifested by the interaction of light and tissue [1]. When illuminating tissue with light, the light will travel along certain paths in the tissue depending on the optical properties [2]. The absorption coefficient determines how far the light propagates in a medium before absorption. The refractive index and the reduced scattering coefficient determine how far the light propagates in the medium before scattering [3]. The absorption is due to different chromophores in the tissue. The chromophores absorb a different amount of light for different wavelength [4]. The scattering is caused by variations in the refractive index within the tissue and is wavelength dependent, which results in a different amount of scattering for different wavelengths [5]. The photons that are scattered and exits the tissue can then be collected by another optical fiber, resulting in a wavelength-dependent reflectance spectrum. The chromophores differ from healthy tissue, tumors and treatment tissues, which results in different reflectance spectra [6].

Various types of optical spectroscopy have been investigated as methods for assessment of tissue pathology [7]. All of these methods have one basic principle in common: the optical spectrum of tissue contains information about the biochemical composition and/or the structure of the tissue, and that information conveys diagnostic information [8].

Optical spectroscopy can also be employed in the management of disease treatment. The diagnostic spectroscopy can be used to monitor response to treatment as well [9].

The benefits of this technique is to develop a diagnostic tool in real-time during surgery and could facilitate the determination of biological changes relevant spectroscopic optical diagnostic techniques for treatment tissue, which would be reduced the number of unnecessary biopsies, the suffering of the patients, and introduce a practical way for surgeons, low cost and ease of implementation, generally mediated with small portable instruments, not requiring any specialized facilities, and eventually not requiring expert interpretation.

\section{Materials and Methods}

Experimental setup for the diffuse reflectance measurements consisted of a pulsed Xenon-arc lamp (HL-2000-Ocean optics) as a broadband light source [10], a spectrometer (USB4000 FLOcean optics) and a fiber probe for the delivery and collection of the light to and from the sample as shown in (Figure 1). The light transport within a tissue a probe that comprises two multimode optical fibers (a source and a detector), which are parallel to each other. 


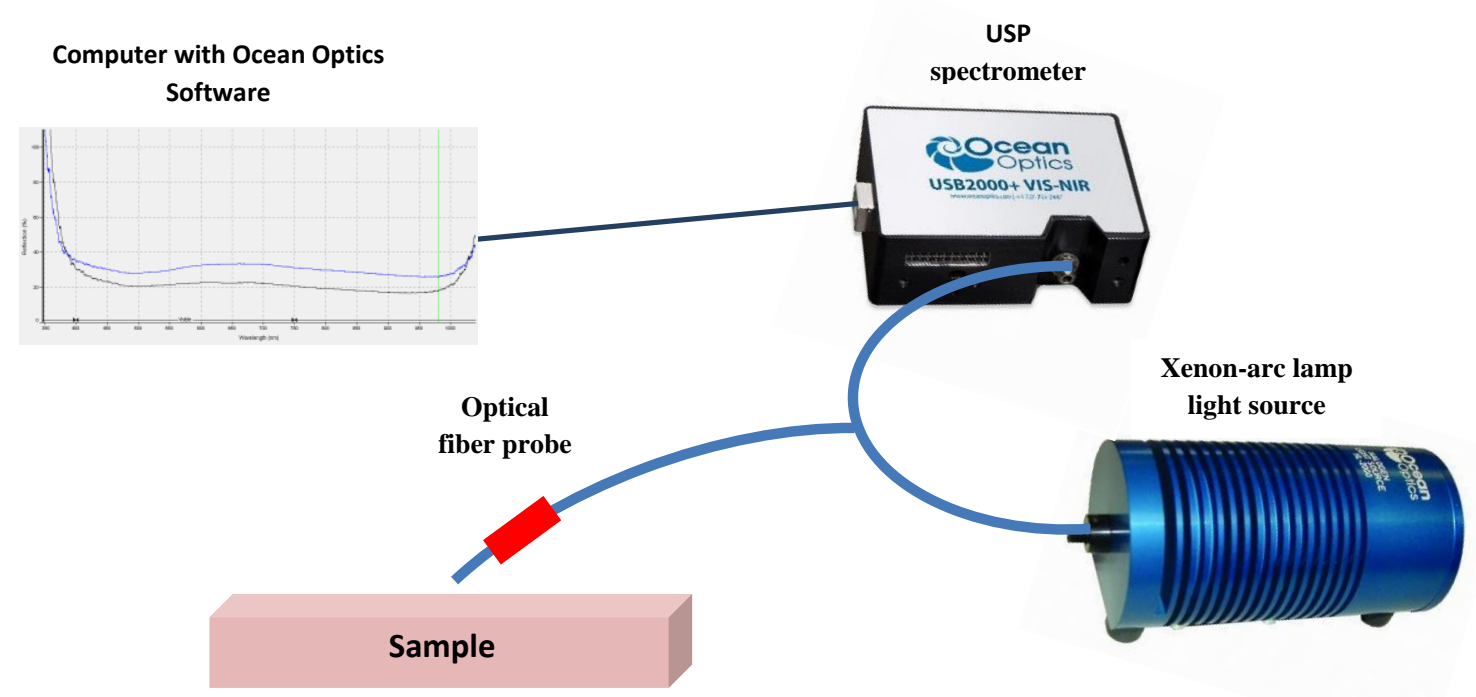

Figure 1. Schematic diagram of the diffuse reflectance spectroscopic system

Each fiber has a core diameter of $200 \mu \mathrm{m}$. Both fibers can be tilted at an angle $(\Theta)$ relative to the tissue surface normal, in the $\mathrm{X}-\mathrm{z}$ plane, such that the fibers are parallel to each other [11]. After the coupling process described in (Figure 1), calibration is initiated by recording the diffuse reflection spectrum of the white standard which is an ideal reflectance at nearly $100 \%$ Reference, by the optical fiber placed on a $45^{\circ}$ angle holder and the holder is in contact with the white standard as shown in (Figure 2). Light from a white light source passes through the optical fiber to the sample, which transmits the reflected light from the sample into the spectrometer connected to a computer to record the spectrum. Always three measurements were taken, which were averaged into one spectrometer reading.

Reflection is the return of radiation by a surface, without a change in wavelength. Reflection can be:

- Specular (the angle of incidence is equal to the angle of reflection)

- Diffuse (the angle of incidence is not equal to the angle of reflection)

Every surface returns both specular and diffuse reflections. Some surfaces may return mostly specular reflection, while others may return mostly diffuse reflection. Specular reflection increases proportionately with the amount of gloss on a surface [12].

Reflection is expressed as a percentage $\left(\% R_{\lambda}\right)$ relative to the reflection from a standard reference substance:

$\% R_{\lambda}=\frac{s_{\lambda}-D_{\lambda}}{R_{\lambda}-D_{\lambda}} * 100 \%$

Eq. 1

Where:

$\mathrm{S}_{\lambda}=$ the sample intensity at wavelength $\lambda$

$\mathrm{D}_{\lambda}=$ the dark intensity at wavelength $\lambda$

$\mathrm{R}_{\lambda}=$ the reference intensity at wavelength $\lambda$

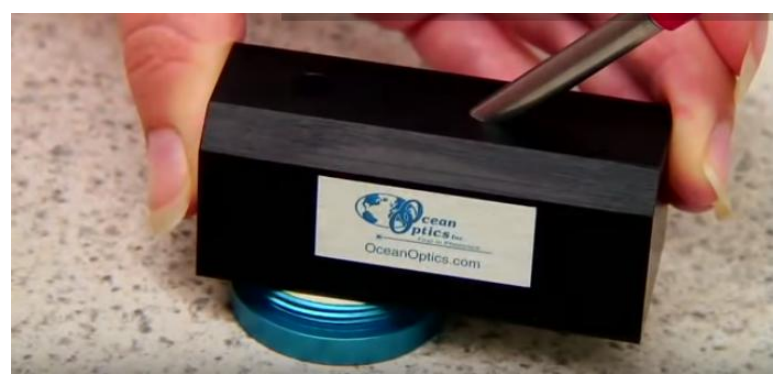

Figure 2. Calibration diffuse reflection spectrum of the white standard at $45^{\circ}$ angle

A light source sends light via the input leg of a reflection probe onto a sample. A reflection probe holder holds the probe in either a 90 or 45 -degree angle from the surface. The output leg of the reflection probe carries light from the sample to the spectrometer, which is connected to the computer $[13,14]$.

\section{Sample preparation}

The experiment on animals happened after institutional ethical approval was obtained, according to Damascus university ethical committee decision no. 3164. Groups of 35 hamsters ranging at 10-12 weeks of age were used. They were housed in good health conditions and at the temperature of the laboratory, where they were given Barley, millet, bran, wheat, maize, and lettuce, as well as water continuously [15]. DMBA was mainly used as a carcinogen in hamster skin [16]. The study area was marked by a permanent ink pen to follow up on the changes in the area identified during the experiment and specimens. The solutions were applied topically on the back of skin area to be studied, $0.5 \mathrm{~W}$ radiation of $980 \mathrm{~nm}$ Diode laser is applied once a week from the time the carcinogen to the end of the experiment. The animals were checked at weekly intervals, and monitored until spontaneous death, or were sacrificed during the experiment. 
The glass slides were cleaned using pure cellulose soft tissue paper wetted with one drop of isopropyl alcohol to remove the impurities placed on the surface, and then immersing these slides in isopropyl alcohol for a minute and wiping them and drying them with hot air.

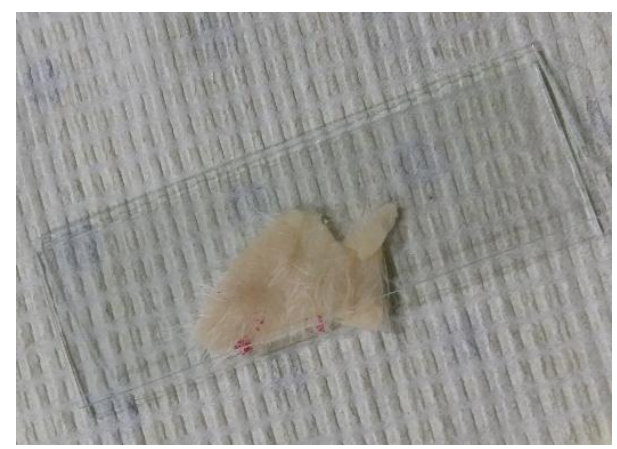

Figure 3. Sample of living tissue on a glass slide

The living tissue was accurately put onto the glass slides as shown in (Figure 3), and then the diffuse reflectance spectrum was taken in the wavelength range of $(400-1000 \mathrm{~nm})$. The sources of uncertainties were maintaining stable, like (light, temperature, and humidity), and uncertainty was well below $\pm 1 \%$. The spectrum was measured in a dark room with ambient laboratory conditions. Also, the calibration procedures were used to normalize variability in the spectral response. The samples were fixed in formalin until the time of diffuse reflectance spectrum measurement.

\section{Results}

Hamsters, in which the 9,10-dimethyl-1, 2-benzanthracene in mineral oil was applied, during the first few weeks following applying carcinogen within $12^{\text {th }}$ week, slight desquamation and minor spots with the light gray color in the area of application and weak hair growth in all the hamster skin observed. Within the $16^{\text {th }}$ week from the beginning of the experiment, numerous minute black spots appeared scattered throughout the treated area. Around the $20^{\text {th }}$ week of the experiment increased pigment lesions with an irregular shape, aggressive and weakness hair growth were observed as shown in Figure 4. The average latency, calculated for all melatonin tumors varied from $12^{\text {th }}$ to $24^{\text {th }}$ weeks see (Table 1). The hamsters were sacrificed week $16^{\text {th }}$.

By recording the diffuse reflectance spectrum in the wavelength range $(400-100 \mathrm{~nm})$ for the samples, a decrease in the diffuse reflectance spectrum during the cancer formation stages from week 12 to the end of the experiment in week 36 was observed (Figure 5).

The groups of hamsters on which the solution of DMBA was applied were exposed to $980 \mathrm{~nm}$ Diode laser of $0.5 \mathrm{~W}$ for $100 \mathrm{~s}$. They showed redness on the surface of the skin after irradiation because of heating of the skin surface, Hyperthermia disappeared two days later and there was no change in the surface of the skin as shown in (Figure 6). When the diffuse reflectance spectrum was recorded during the experiment, a decrease in the spectrum was observed starting from week 28, indicating a delay in the carcinogen see (Figure 7).

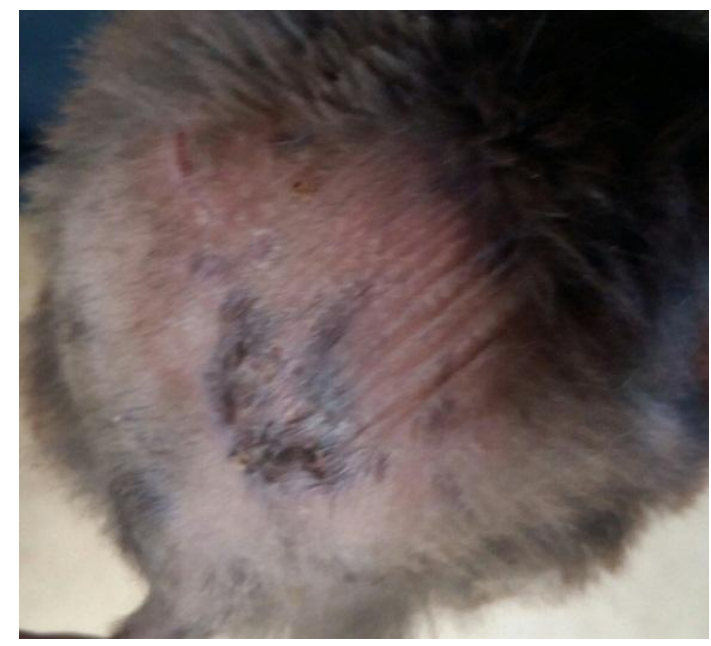

Figure 4. $20^{\text {th }}$ week, increased pigment lesions with an irregular shape, aggressive

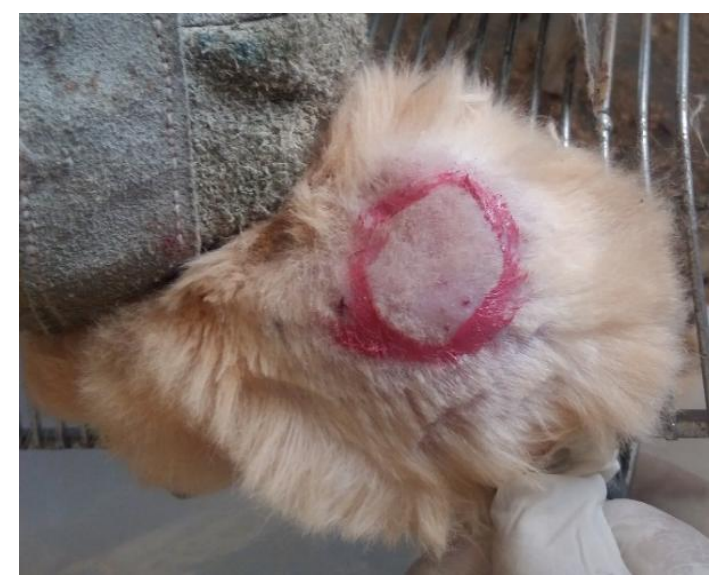

Figure 6. Application of the solution of DMBA and $0.5 \mathrm{~W}$ irradiation to $980 \mathrm{~nm}$ Diode laser $0.5 \mathrm{~W}$

Table 1. Results of application of carcinogen on hamster skin

\begin{tabular}{|c|c|c|c|}
\hline $\begin{array}{c}\text { No. } \\
\text { Weeks }\end{array}$ & DMBA Control & $\begin{array}{l}980 \mathrm{~nm} \text { Diode } \\
\text { Laser Control } \\
\end{array}$ & $\begin{array}{c}\text { DMBA+ } 980 \mathrm{~nm} \\
\text { Diode Laser } 0.5 \mathrm{~W} \\
\end{array}$ \\
\hline 12 & $\begin{array}{l}\text { light pigmented lesions, } \\
\text { weak hair growth }\end{array}$ & $\mathrm{N}$ & $\mathrm{N}$ \\
\hline 16 & $\begin{array}{c}\text { increased pigmented } \\
\text { lesions, weak hair growth }\end{array}$ & $\mathrm{N}$ & $\mathrm{N}$ \\
\hline 20 & $\begin{array}{l}\text { increased pigment lesions, } \\
\text { aggressive, and weak hair } \\
\text { growth }\end{array}$ & $\mathrm{N}$ & $\mathrm{N}$ \\
\hline 24 & $\begin{array}{l}\text { increased pigment lesions, } \\
\text { aggressive, and weak hair } \\
\text { growth }\end{array}$ & $\mathrm{N}$ & $\mathrm{N}$ \\
\hline 28 & $\begin{array}{c}\text { pigment lesions, } \\
\text { aggressive, and weak hair } \\
\text { growth }\end{array}$ & $\mathrm{N}$ & $\mathrm{N}$ \\
\hline 32 & $\begin{array}{c}\text { pigment lesions, } \\
\text { aggressive, and weak hair } \\
\text { growth }\end{array}$ & $\mathrm{N}$ & $\mathrm{N}$ \\
\hline 36 & $\begin{array}{c}\text { pigment lesions, } \\
\text { aggressive, and weak hair } \\
\text { growth }\end{array}$ & $\mathrm{N}$ & $\mathrm{N}$ \\
\hline
\end{tabular}




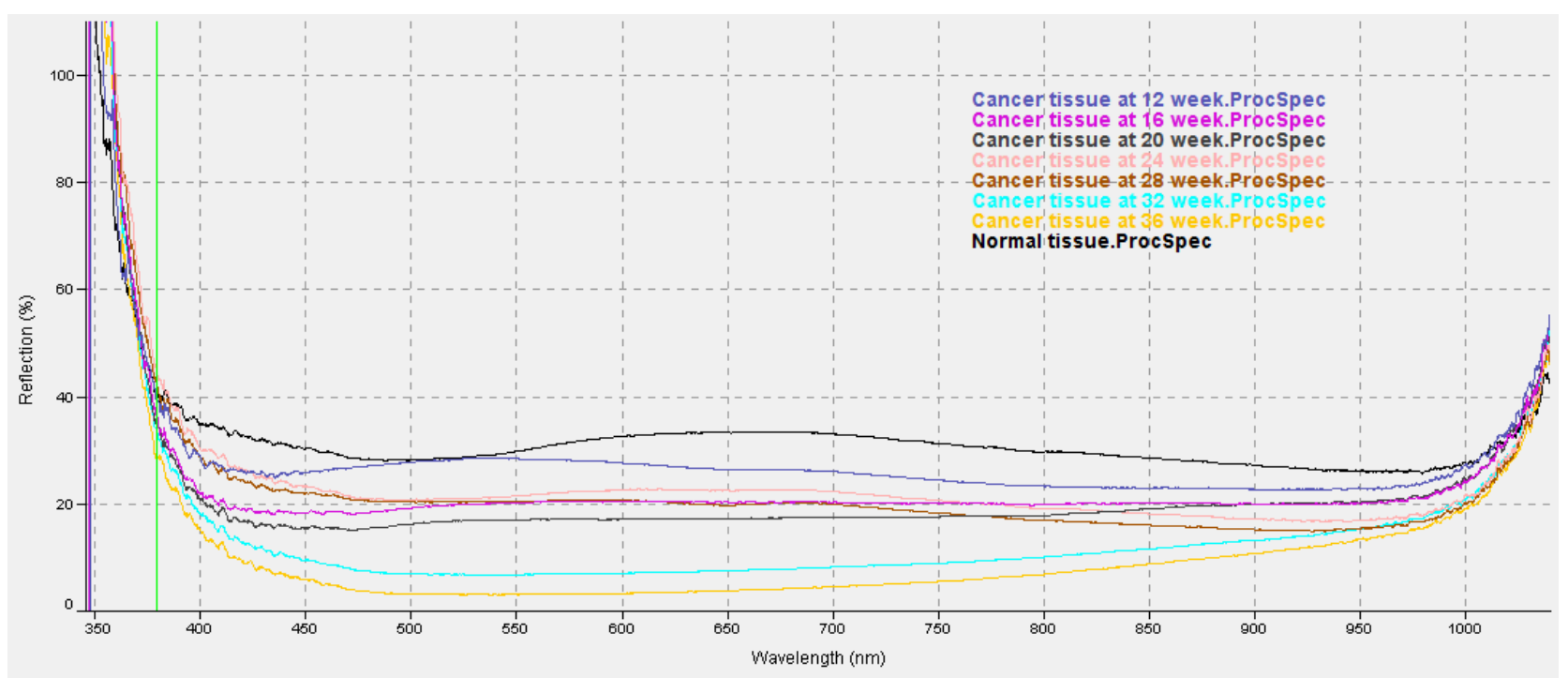

Figure 5. The diffuse reflectance spectrum at $400-1000 \mathrm{~nm}$ for all changed occurred on tissue during carcinogen transformation stages

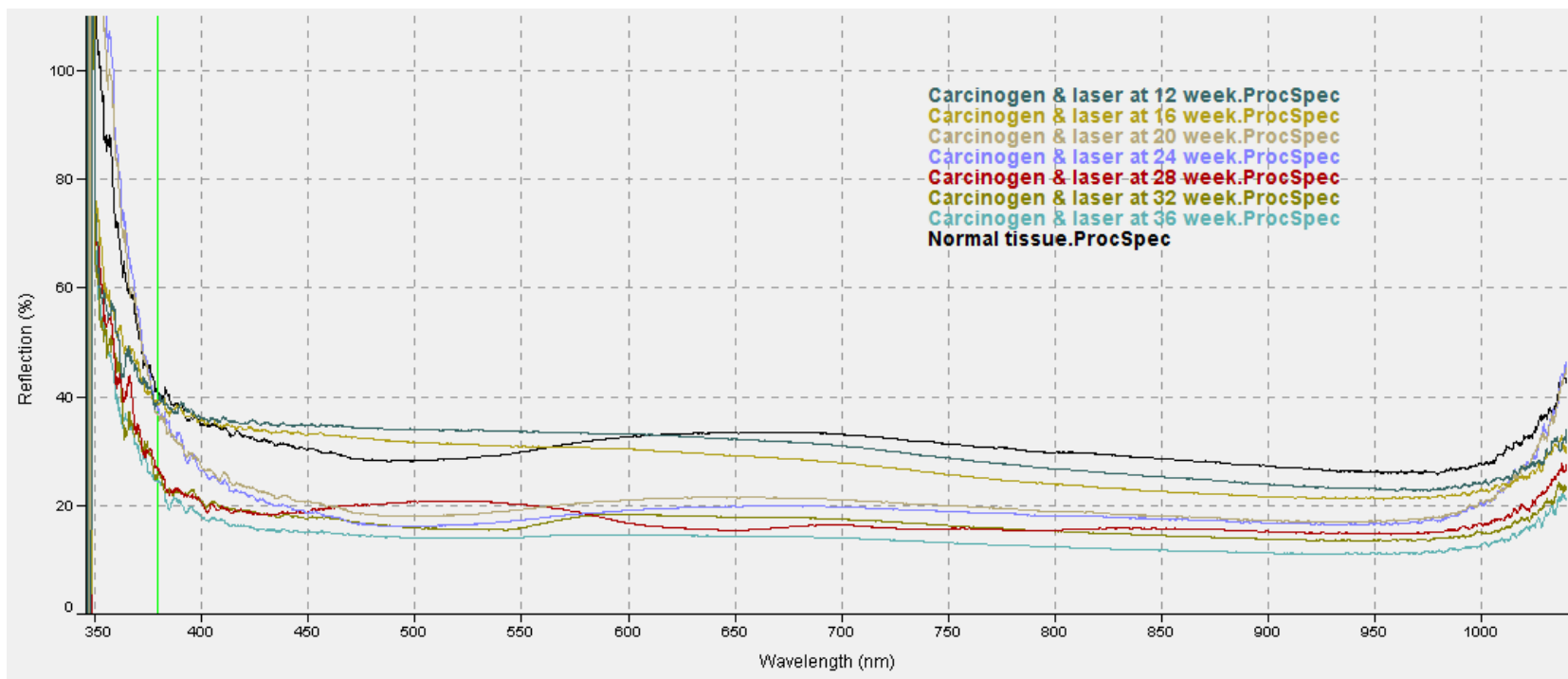

Figure 7. The diffuse reflectance spectrum of hamsters which are applied the solution of DMBA and were exposed to $980 \mathrm{~nm}$ Diode laser 0.5 W

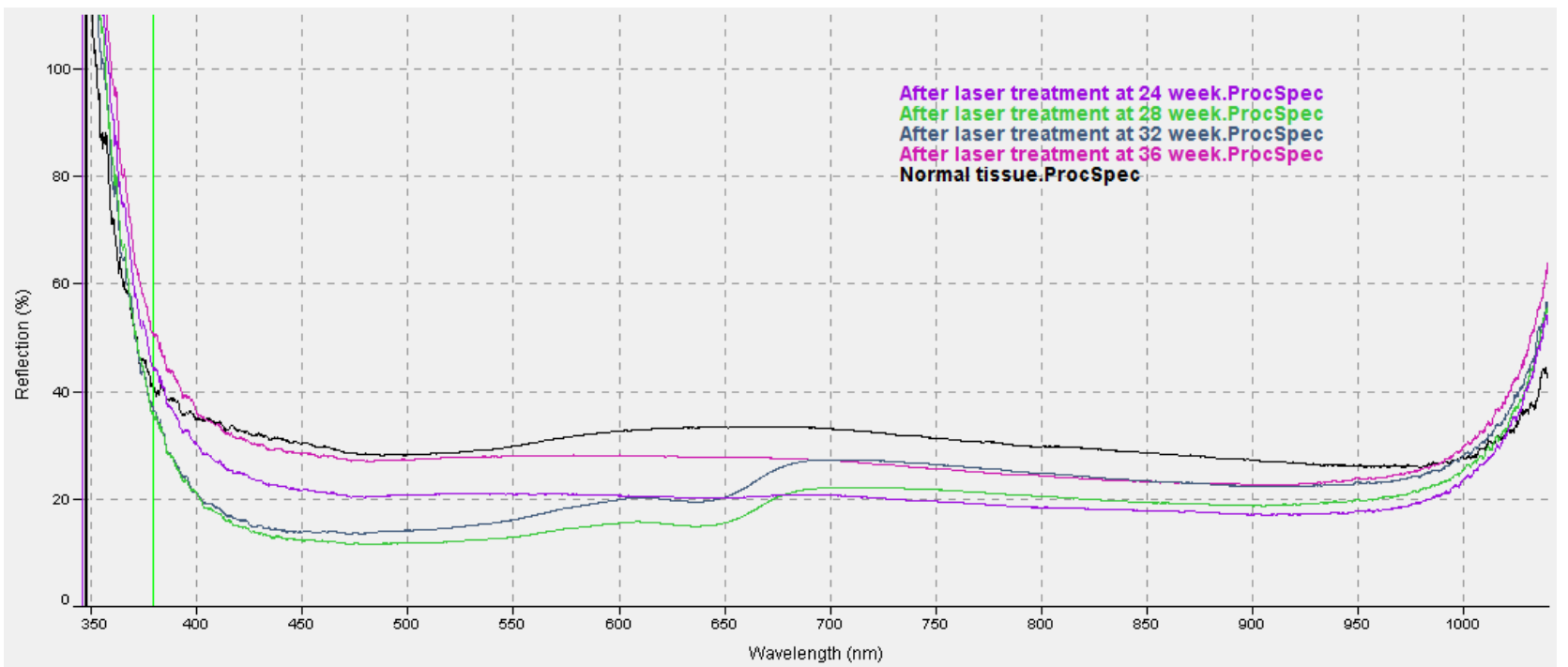

Figure 9. The diffuse reflectance spectrum of hamster's samples, which were exposed to 98 0nm Diode laser 0.5 W after the development of melanoma 
Table 2. A comparison of the diffuse reflectance spectral ratios between samples that were applied the carcinogens only and samples that were exposed to the laser during the carcinogen

\begin{tabular}{|c|c|c|c|c|c|c|}
\hline \multirow{3}{*}{ No. Weeks } & \multicolumn{6}{|c|}{ Reflection $\left(\mathbf{R}_{\lambda} \%\right)$} \\
\hline & \multicolumn{2}{|c|}{$\lambda=450 \mathrm{~nm}$} & \multicolumn{2}{|c|}{$\lambda=700 \mathrm{~nm}$} & \multicolumn{2}{|c|}{$\lambda=980 \mathrm{~nm}$} \\
\hline & DMBA & With laser & DMBA & With laser & DMBA & With laser \\
\hline 12 & $25.37 \%$ & $29.70 \%$ & $25.92 \%$ & $32.8 \%$ & $24 \%$ & $26 \%$ \\
\hline 16 & $23.24 \%$ & $29.8 \%$ & $22.21 \%$ & $35.3 \%$ & $17.96 \%$ & $27 \%$ \\
\hline 20 & $21.72 \%$ & $33.6 \%$ & $19.84 \%$ & $32.35 \%$ & $16.75 \%$ & $23.9 \%$ \\
\hline 24 & $18.13 \%$ & $32.9 \%$ & $20 \%$ & $28.2 \%$ & $21.04 \%$ & $22.07 \%$ \\
\hline 28 & $15.29 \%$ & $20.56 \%$ & $17.04 \%$ & $21 \%$ & $20.84 \%$ & $18.7 \%$ \\
\hline 32 & $9.5 \%$ & $18.36 \%$ & $8.13 \%$ & $19.57 \%$ & $17.45 \%$ & $17.16 \%$ \\
\hline 36 & $5.08 \%$ & $17.5 \%$ & $4,45 \%$ & $17.3 \%$ & $15.55 \%$ & $13.08 \%$ \\
\hline Normal Tissue & \multicolumn{2}{|c|}{$30.28 \%$} & \multicolumn{2}{|c|}{$32.94 \%$} & \multicolumn{2}{|c|}{$25.72 \%$} \\
\hline
\end{tabular}

A group of hamsters was isolated after the appearance of melanoma in the $20^{\text {th }}$ week. The application of carcinogens stopped and the hamsters were exposed to Diode $980 \mathrm{~nm}$ laser $0.5 \mathrm{~W}$ once a week for 8 weeks, resulting in reducing the size and color of dark spots as shown (Figure 8). Diffuse reflectance spectrum showed an increase until the end of the experiment, indicating an improvement in skin tissue and the laser effect on the melanoma cured (Figure 9). Table 1 shows the results of the application of carcinogen on hamster skin from week 12 to 36 .

By studying the diffuse reflectance spectrum mentioned in this paper, we note that the best area where changes are clearly shown is in the near infra-red region where changes are obvious. So Table 2 shows a comparison of the diffuse reflectance spectral ratios at three wavelengths of the hamster samples which applied the carcinogen only and the samples that were exposed to the laser during the application of the carcinogen, the changes are very obvious from healthy to cancer tissue at the end of experiment as shown in Table 2, which is demonstrates these differences between 7 periods covering the tissue transformation from week 12 to week 36 . Table 3 shows an increase in diffuse reflectance spectral ratios indicating an improvement in skin tissue and the melanoma cured.

\section{Discussion}

A Diffuse Reflectance Spectrum recorded from biological tissue is composed of two components: absorption and scattering. As for scattering alone, the scattering is inversely proportional to wavelength, and according to Rayleigh and Mie scattering, as found by G. Einstein et al. [17], the extracted scattering component from Diffuse Reflectance Spectroscopy recorded from excised oral tissue, showed that the decay of scattering has a smooth curve with no specific features. This trend of scattering over the wavelength range applies to epithelium tissue as well as skin tissue in both humans and animals.

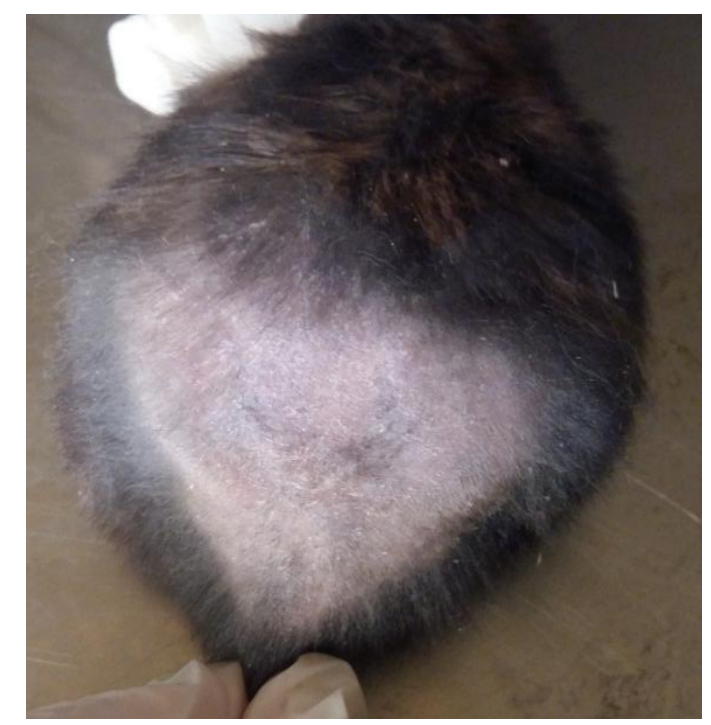

Figure 8. Week 24, reducing the size and color of dark spots after stopping carcinogen and laser irradiation of $0.5 \mathrm{~W}$

Table 3. Diffuse reflectance spectral ratios showed an increase until the end of the experiment

\begin{tabular}{cccc}
\hline \hline \multirow{2}{*}{ No. weeks } & \multicolumn{3}{c}{ Reflection $\left(\mathbf{R}_{\boldsymbol{\lambda}} \boldsymbol{\%}\right)$} \\
\cline { 2 - 4 } & $\boldsymbol{\lambda}=\mathbf{4 5 0} \mathbf{~ n m}$ & $\boldsymbol{\lambda}=\mathbf{7 0 0} \mathbf{~ n m}$ & $\boldsymbol{\lambda}=\mathbf{9 8 0} \mathbf{n m}$ \\
\hline 24 & $12.15 \%$ & $21.9 \%$ & $21.8 \%$ \\
28 & $13.66 \%$ & $27.21 \%$ & $24.43 \%$ \\
32 & $21.41 \%$ & $21.59 \%$ & $19.9 \%$ \\
36 & $28.20 \%$ & $27.21 \%$ & $25.6 \%$ \\
\hline Normal tissue & $32.94 \%$ & $25.72 \%$ & $32.94 \%$ \\
\hline \hline
\end{tabular}

Likewise, the results in our work (DRS spectra) are consistent with the above findings. That is, for the sample used in the presented manuscript, there is no contribution of absorption to the recorded reflection due to lack of hemoglobin and the remaining contribution is only to scattering effect. However, the presented work focused on different stages of tissue transformation not only the extreme cases (healthy and cancer). This can be explained by the subtle differences between the corresponding spectra. Yet, these subtle differences are good enough to differentiate between these stages. 


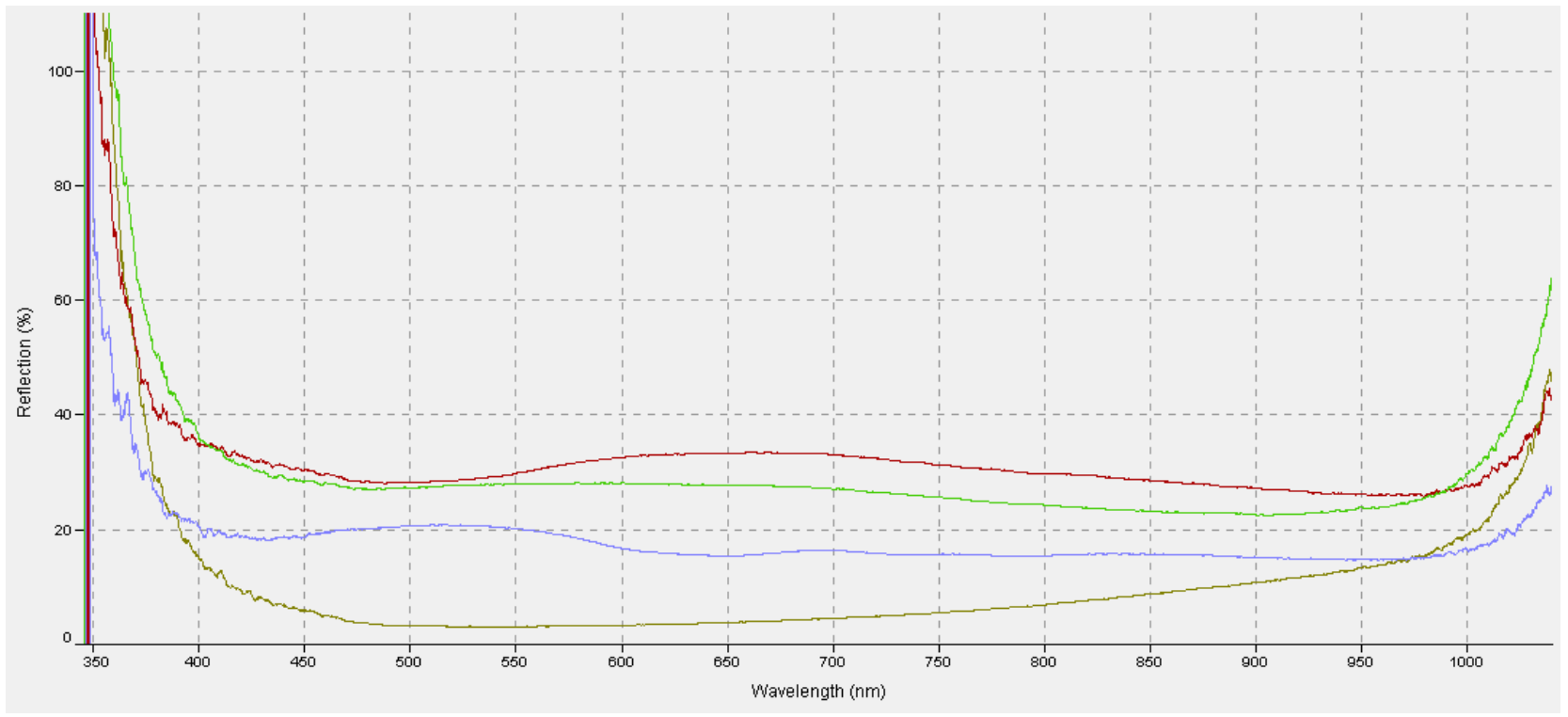

Figure 10. A comparison of the diffuse reflectance spectral ratios between samples at the end of the experiment

The effects of $980 \mathrm{~nm}$ Diode laser while applying the carcinogenic chemicals DMBA on the skin of the hamster and their diffuse reflectance spectrum have been studied. The hamsters which were not irradiated by laser consistently gave rise to melatonin tumors and there was a decrease in the diffuse reflectance spectrum during the cancer formation stages until the end of the experiment in week 36 was observed. The hamsters on which the solution of DMBA was applied and exposed to diode $980 \mathrm{~nm}$ laser irradiation power of $0.5 \mathrm{~W}$ did not show any change in the skin, and a marked decrease in diffuse reflectance spectrum was observed starting from week 28 , indicating a delay in the carcinogenesis.

For the hamster which we stopped applying a carcinogen, and exposed to the Diode $980 \mathrm{~nm}$ laser power $0.5 \mathrm{~W}$, the diffuse reflectance showed a noticeable increase until the end of the experiment, indicating an improvement in skin tissue and the laser effect on the melanoma cured. It was noted that all the changes in the studied tissue were evident in the infrared range, so we recommend recording the diffuse reflectance spectrum in this region.

For comparison and summary, the results, Figure 10 and Table 4 show the changes in diffuse reflectance spectrums in the specimens studied clearly at week 36 at the end of the experiment, and their diffuse reflectance spectral ratios at $(750$, $800,850) \mathrm{nm}$ in the infrared region.
Table 4. A comparison of the diffuse reflectance spectral ratios for the specimens at the end of the experiment in the infrared region

\begin{tabular}{cccc}
\hline \hline \multicolumn{4}{c}{ Reflection (R\%) } \\
\hline Wavelength & $\mathbf{7 5 0} \mathbf{~ n m}$ & $\mathbf{8 0 0} \mathbf{~ n m}$ & $\mathbf{8 5 0} \mathbf{~ n m}$ \\
\hline Normal tissue & $31.15 \%$ & $29.65 \%$ & $28.46 \%$ \\
After laser treatment & $25.51 \%$ & $24.14 \%$ & $23.11 \%$ \\
DMBA \& laser & $12.98 \%$ & $12.23 \%$ & $11.60 \%$ \\
Carcinogen tissue & $5.41 \%$ & $6.76 \%$ & $8.67 \%$ \\
\hline \hline
\end{tabular}

\section{Conclusion}

The aim of this paper is to demonstrate a diagnostic tool that can be used to determine the optical spectrum of early changes of pre-cancer and cancer using Diffuse Reflectance Spectroscopy. Optical techniques are currently being used to identify changes that occur in biological tissues associated with disease progression. It is simple in concept, requires relatively straightforward and low-cost instrumentation and has the potential to give an immediate diagnosis without the need to remove tissue from the patient or for the result to be interpreted by an expert pathologist.

The diffuse reflectance spectrum of samples showed that the $980 \mathrm{~nm}$ diode laser had a significant role in delaying the onset of cancer, and reducing the size of cancer legions and improving the appearance of skin.

Future work can be carried out to validate these optical techniques and to apply them in clinical settings. 


\section{References}

[1] Volynskaya Z, Haka AS, Bechtel Kl, et al. Diagnosing breast cancer using diffuse reflectance spectroscopy and intrinsic fluorescence spectroscopy. J Biomed Opt. 2008;13(2):024012-024012-9.

[2] Bigio IJ, Bown SG. Spectroscopic sensing of cancer and cancer chemotherapy, current status of translational research. Cancer Biol Ther. 2004;3(3):259-267.

[3] Flock ST, Jacques SL, Wilson BC, et al. Optical Properties of Intralipid:A Phantom Medium for Light Propagation Studies. Lasers Surg Med. 1992;12(5):510-519.

[4] Andersson A. Look-up table based Monte Carlo inverse model as a tool to discover liver tumors. Bachelor thesis. Lund University, 2015.

[5] Reif R, A'Amar O, Bigio IJ. Analytical model of light reflectance for extraction of the optical properties in small volumes of turbid media. Appl Opt. 2007;(46)29:7317-7328.

[6] van Veen RL, Amelink A, Menke-Pluymers M, et al. Optical biopsy of breast tissue using differential path-length spectroscopy. Phys Med Biol. 2005;50(11):2573-2581.

[7] Bigio IJ, Mourant JR. Optical Biopsy. In: Encyclopedia of Optical Engineering. Marcel Dekker Press, 2003.

[8] Alhamami M. Photoacoustic Detection and Optical Spectroscopy of High-Intensity Focused Ultrasound-Induced Thermal Lesions in Biologic Tissue. Master thesis. Ryerson University, 2013.

[9] Jacques SL. Corrigendum: Optical properties of biological tissues: a review. Phys Med Biol. 2012;58:5007.

[10] Yaroslavsky AN, Schulze PC, Yaroslavsky IV, et al. Optical properties of selected native and coagulated human brain tissues in vitro in the visible and near infrared spectral range. Phys Med Biol. 2002;47(12):2059-2073

[11] Friebel M, Roggan A, Mueller G, Meinke M. Determination of optical properties of human blood in the spectral range 250 to 1100 nm using Monte Carlo simulations with hematocrit-dependent effective scattering phase functions. J Biomed Opt. 2006;11(3):34021.

[12] Romanos GE, Henze M, Banihashemi S, et al. Removal of epithelium in periodontal pockets following diode (980 $\mathrm{nm})$ laser application in the animal model: an in vitro study. Photomed Laser Surg. 2004;22(3):177-183.

[13] Anderson RR. Lasers in Dermatology - A Critical Update. J Dermat. 2000;27(11):700-705.

[14] Sozzi M, Fornaini C, Cucinotta C, et al. Dental ablation with 1064 nm, 500 ps, Diode pumped solid state laser: A preliminary study. Laser Ther. 2013;22(3)195-199.

[15] Sugimura T. Studies on environmental chemical carcinogenesis in Japan. Science. 1986;233(4761):312-318.

[16] Shubik P, Pietra G, Della Porta G. Studies of Skin Carcinogenesis in the Syrian Golden Hamster. Cancer Res. 1960;20:100-105.

[17] Einstein G, Udayakumar K, Arun PR, et al. Diffuse reflectance spectroscopy for monitoring physiological and morphological changes in oral cancer. Optik. 2016;127(3):1479-1485. 